"It is timely for us to be far more proactive in providing smoking cessation advice to our patients."

\section{Smoking success}

That it is good to go away but also good to come home is a maxim expressed in many ways and in many languages and cultures. The recent bans on smoking in the constituent parts of the UK make that even more pertinent, especially when returning from a country where no such restrictions apply.

To come back to London, perhaps not one of the cleanest air environments in the world, and to be reminded of how pleasant it is now to be able to eat, drink and to just 'be' in a nonsmoking public environment is a complete joy. Colleagues (that is non-smoking colleagues) in Scotland and Wales have been able to experience this for longer than we have in England but already, like the wearing of seat belts in cars, it now seems incredible that we ever allowed such a pernicious habit to have free reign.

Pleasing as it is for the general ambiance, how much more positive to read from recently published studies that the ban is already starting to show health gains. In Wales a mere six months after their ban came into being it is estimated that 200 non-smoking lives have already been saved. While in Scotland a University of Glasgow study has found a 17 per cent fall in admissions for heart attacks in the first year after the smoking ban came into force.

With so much attention now being drawn to the subject and with such positive outcomes, it is timely for us to be far more proactive in providing smoking cessation advice to our patients. As an element of health education which we hardly if ever formerly broached it is now fast becoming a standard. Apart from other benefits the approach highlights the links between oral health and general health, notably in periodontal conditions but also in other soft tissue pathologies as well as tooth staining, bad breath and post-operative healing.

Surveys of dentists have previously found that about twothirds feel it is part of their role to discuss smoking cessation with patients, and there is evidence that the percentage of GDPs who ask patients about their smoking habits is steadily increasing. Two initial reservations are frequently offered: that it is an area of personal choice on behalf of the patient into which we have no business enquiring, and that whatever we might say is hardly likely to make a difference anyway. Neither objection stands up to scrutiny. In terms of personal habits we have no such reservations when it comes to advising on diet and on oral hygiene, yet discussing either could be said to be equally invasive since they are frequently based on personal choice. Importantly, all have a direct bearing on the quality of general health and wellbeing.
Secondly, even the briefest mention of smoking by a health professional, including ourselves, has a powerful effect. For instance, it has been calculated that three minutes spent advising a patient to stop will encourage 2 per cent to abstain for at least six months. This may not seem like a large effect but when applied to actual numbers, given that 120,000 people die prematurely in the UK every year due to smoking related diseases, 2 per cent equates to a significant fall, especially if we all provided such a service starting today. ${ }^{1}$

Lack of training is another commonly expressed concern but a variety of sources now exists for courses and literature for the dentist and other team members. A popular system is that of using the four (or sometimes five) 'A's; Ask, Advise/Assess, Arrange, and Assist. In this process, the recent smoking bans have had a synergistic effect by providing essential support for the smoker who wants to quit. The All Wales Smoking Cessation Service's report, published to coincide with the six month anniversary of the ban, shows a 20 per cent increase in people asking for help to give up smoking since the legislation came into force. Of the 9,375 people who took part in a treatment programme, 4,757 reported they had given up smoking.

Support on a wider basis is important to the success of individual attempts for two reasons. Few smokers manage to quit at their first attempt meaning that they need continued nonjudgmental support on a regular basis. Also, the reasons for lack of success often hinge around the temptation to indulge again on occasions when peer pressure can be applied, such as in pubs and restaurants or at social gatherings. The bans mean that there are fewer opportunities to succumb to such provocation, which in turn is likely to increase the success rate of attempts to quit.

As we become further involved with lifestyle choices and changes in behaviour in relation to our patients' wishes and the work that we do, it is inevitable that smoking cessation will become a more central aspect of our services. With this month's Mouth Cancer Awareness campaigns as a spur it is the ideal opportunity for us to all quit as well. Quit smoking ourselves and quit overlooking this important element of patient health for which we owe a responsibility of care.

Stephen Hancocks OBE Editor-in-Chief

1. Watt R, Beaglehole R H. Helping smokers stop. A guide for the dental team. London: Health Development Agency, 2004.

DOI: $10.1038 / b d j .2007 .1039$ 